\title{
Modeling mass transfer in fracture flows with the time domain-random walk method
}

\author{
J. Kuva ${ }^{1}$ (D) M. Voutilainen ${ }^{2} \cdot$ K. Mattila $a^{3,4}$ \\ Received: 2 May 2018 / Accepted: 21 June 2019 / Published online: 9 July 2019 \\ (C) The Author(s) 2019
}

\begin{abstract}
The time domain-random walk method was developed further for simulating mass transfer in fracture flows together with matrix diffusion in surrounding porous media. Specifically, a time domain-random walk scheme was developed for numerically approximating solutions of the advection-diffusion equation when the diffusion coefficient exhibits significant spatial variation or even discontinuities. The proposed scheme relies on second-order accurate, central-difference approximations of the advective and diffusive fluxes. The scheme was verified by comparing simulated results against analytical solutions in flow configurations involving a rectangular channel connected on one side with a porous matrix. Simulations with several flow rates, diffusion coefficients, and matrix porosities indicate good agreement between the numerical approximations and analytical solutions.
\end{abstract}

Keywords Matrix diffusion $\cdot$ Advection $\cdot$ Porous media $\cdot$ Solute transport $\cdot$ Breakthrough curve $\cdot$ Simulation

\section{Introduction}

Intracellular motion of biomolecules, propagation of contaminants, plant nutrients in soils, and migration of radionuclides within a geosphere are some examples of phenomena involving particle transport due to advection and diffusion. The related modeling tasks vary in difficulty from nearly trivial to particularly challenging. When the transport occurs in simple domains and involves constant transport parameters, strictly analytical modeling is possible. However, only a handful of more complex cases allow purely analytical treatment; beyond these cases, computational modeling is necessary.

$\triangle$ J. Kuva

jukka.kuva@iki.fi

M. Voutilainen

ma.voutilainen@helsinki.fi

1 Geological Survey of Finland, Espoo, Finland

2 Department of Chemistry, University of Helsinki, Helsinki, Finland

3 Department of Physics, University of Jyväskylä, Jyväskylä, Finland

4 Laboratory of Physics, Tampere University of Technology, Tampere, Finland
Here, we focus on the computational modeling of mass transfer in fracture flows complemented with matrix diffusion $[3,12,27]$. The overall transport can be illustrated as follows: (1) solute (pulse) is released into the fracture where it is transported by advection and diffusion; (2) during propagation along the fracture, a (small) portion of the solute enters the porous media making it effectively a solute reservoir; (3) solute is (slowly) transported by diffusion within the porous media; and (4) solute returns gradually from the porous media back into the fracture, thus completing the cycle (a secondary release occurs). An arbitrary solute particle can undergo steps 2 to 4 multiple times. Thus, when compared with pure fracture flow, the matrix diffusion introduces a retardation effect on the solute transport. Specifically, the solute breakthrough curves now exhibit longer tails. The effect is even more pronounced with sorption onto mineral surfaces inside the porous media or at the fracture perimeters [41]. To provide a specific example, the safety of the final repository for spent nuclear fuel in Finland [33] has driven the development of modeling capabilities for the phenomena described above.

Modeling mass transfer in fracture flows together with matrix diffusion is challenging because of the multiple temporal and spatial scales inherently present in the phenomena. Specifically, these include fast transport in fractures alongside slow transport inside the porous media, in this case rock. Alternatively, fractures with a relatively 
large diameter, possibly constituting a complex networktype flow system, are in contact with much smaller pores thus forming highly tortuous transport pathways through the rock. The challenge remains even with the common simplification where the flow field responsible for advection is assumed to be independent of the solute concentration and, moreover, fully developed prior to the initial release. Although modeling can be aided by simplified laboratory and in situ experiments [20, 50, 51], which on occasion can be treated analytically [17, 18, $43,48]$, in reality the fractures or flow channels are complex and the surrounding rock is typically heterogeneous. While these more realistic systems call for computational modeling, such modeling also entails significant challenges.

The multiple spatial scales involved generally inflict a large burden even for contemporary computational resources. The standard remedy is to treat the rock as a heterogeneous bulk material with spatially varying transport coefficients, for example, the effective diffusion coefficient or permeability; here, the ab initio approach of modeling rock at the pore scale is abandoned. The usually low rock porosity implies small values for the transport coefficients when compared with the corresponding values in the fracture. Such values may even be several orders of magnitude smaller. The values of the transport coefficients usually have a discontinuity at the interface between the fracture and the surrounding rock. Furthermore, within the rock, there may be significant spatial variation, or even discontinuities, in the transport coefficients due to distinct minerals with sharp interfaces and changes in rock composition or type. Highly heterogeneous transport coefficients with discontinuous can defeat computational methods; in these cases enforcing stable simulations, while accurately tracking the fast dynamics, require refined time resolution, which then implies extremely laborious time evolution for capturing the essential slow dynamics.

In recent studies, several alternative means have been developed to overcome the computational challenges discussed above. McDermott et al. [25] proposed a hybrid analytical-numerical modeling method, where the transport in fractures is resolved by numerical means and the matrix diffusion is approximated analytically, thus avoiding the excessively lengthy simulations otherwise necessary. However, resorting to analytical treatment unavoidably introduces further assumptions and simplifications, which limit the scope of the approach. Toivanen et al. [45] adopted the classical random walk method for a purely computational modeling of transport, also with sorption [46]. Here, the solute transport is modeled by tracking tracer particles obeying stochastic dynamics. As the random walk method does not suffer from stability issues in the usual sense, there seems to be more freedom in choosing a convenient time resolution. However, to properly enforce the prescribed stochastic particle dynamics, a rather fine time resolution is still necessary and places a heavy strain on the computational resources.

The time domain-random walk (TDRW) method was originally developed for diffusive mass transfer in heterogeneous media with locally known transport coefficients $[6,7,24]$. In the TDRW method, tracer particles obeying stochastic dynamics are tracked. Specifically, the particles jump between sites of a regular grid, or a lattice, and both the direction and duration of a jump are random variables. Moreover, a local probability is assigned for each jump direction in such a manner that, for example, a jump into a neighboring, low porosity site is less likely than a jump into a higher porosity site. The constant transition time characterizing the standard random walk method is effectively replaced with a variable transition time facilitating, in principle, computationally efficient simulations. One possible difficulty is that to gather sufficient statistics also from the low porosity regions, tracking a relatively larger number of tracer particles may be necessary. This is an aspect highly dependent on the system configuration. It is noteworthy that modern computational hardware relies increasingly on parallel processing; pushing the computational burden into the number of particles thus appears advantageous.

The TDRW method has been used to study the effect of centimeter-scale structural heterogeneities on diffusion in various cases [36, 37, 40, 49]. The TDRW method has also been used to model transport in fractured media $[28,29]$ and has been shown to be a powerful tool for analyzing results of in situ and laboratory experiments, especially when initial or boundary conditions are complex [1, 15, 16, 49]. Previously, Dentz et al. [9] modified the TDRW method to include sorption by using specific functions for trapping frequency and number of trapping events. Voutilainen et al. [52] developed this idea further so that the parameter typically measured for rate of sorption (distribution coefficient) can be utilized. Only very recently, the TDRW method was extended to also include advection [13, 38]; a first-order accurate upwind (finite-difference) approximation of the advective massflux was proposed. Although this particular approximation provides unconditionally positive jump probabilities (cf. unconditional stability), it is known in the context of finite-difference and finite-volume methods that first-order upwind schemes introduce severe numerical diffusion, especially when simulating configurations involving large concentration gradients. Therefore, simulation of fracture flows together with, for example, tracer pulses would be problematic.

As a related matter, the TDRW method can be viewed as a reincarnation of the lattice kinetic Monte Carlo (LKMC) method. There does not appear to be a definite point of 
origin for LKMC, but early formulations can be found from Refs. [26] and [4]. Only recently has Flamm et al. [11] extended the LKMC method to include advection; their proposal is essentially the same as that proposed in Refs. $[13,38]$. Lee and Sinno [22] very recently revisited this scheme and proposed an improvement constructed starting from a microscopic premise. Here, the flux terms are computed using an approximation known as the exponential scheme in the finite-volume context. Based on the literature, the difference between the TDRW and LKMC methods, if any, is that the LKMC method advocates the microscopic perspective, both in development and application of the method, while TDRW similarly favors the macroscopic perspective.

Regarding the currently considered transport modeling, similar problems in a larger scale have previously been studied using methods such as discrete fracture network (DFN) modeling [5, 10, 53], continuous time random walks $[2,8,30]$, and multi-rate mass transfer modeling [14, 44]. In general, the large-scale models rely on various mathematical functions with case-dependent parameters that depict the transport in the fractures and interaction of solutes in the fracture and porous matrix. In addition to the TDRW approach, systems with small-scale heterogeneities have also recently been studied with a micro-continuum approach [47] and micro-DFN modeling [42]. In small-scale systems, it is possible to model the interaction of solutes in the fracture and porous matrix explicitly and thus the effects arising from, for example, heterogeneity can be directly observed. Typically, it is challenging to up-scale the results of small-scale approaches to field scale. However, it is possible to validate the mathematical expressions of largescale methods by applying the results from small-scale approaches.

A systematic derivation of TDRW schemes is presented, starting from the macroscopic mass-balance equations (a top-down derivation). The advection-diffusion model considered is first established in Section 2. Then, in Section 3, a corresponding, second-order accurate TDRW scheme is derived and proposed. This advance to stateof-the-art is relevant for two reasons. Firstly, the accuracy of the advective and diffusive flux approximations is now of the same order and, secondly, the adopted centraldifference approximation of the advective flux is not prone to numerical diffusion. These advantages are balanced with a disadvantage. Due to utilization of the central-difference approximation, a local condition for the grid Péclect number is introduced to ensure strictly positive jump probabilities. Specifically, a restriction on the simulation parameters is imposed (analogous to a stability condition). When this condition is fulfilled, the proposed TDRW scheme provides accurate solutions for advection-diffusion problems as demonstrated in Section 4, where comparisons between numerical and analytical solutions for several benchmark cases are presented. Furthermore, when the condition is fulfilled, the central-difference scheme produces more reliable results than the first-order accurate upwind scheme. Conclusions are presented at the end of this article.

\section{Advection-diffusion model}

The focus in this work is on modeling mass transfer in fracture flows involving matrix-diffusion. To this end, a porous material saturated by a fluid (or solvent) which, in turn, carries tracer (or solute) particles, is considered. The general mass-balance equation serves as the starting point; the (volumetric) solute concentration field $c$ evolves according to

$\partial_{t} c+\nabla \cdot \mathbf{j}_{p}=0$

where $\mathbf{j}_{p}$ is the mass-flux of the solute within the pore system. This total mass-flux is then decomposed into contributions due to advection and diffusion. The diffusive part is modeled using

$\mathbf{j}_{p, d}=-\epsilon D_{p} \nabla c_{p}$,

where $c_{p}=c / \epsilon$ is the concentration in the solvent and $\epsilon$ is the local porosity of the medium (between 0 and 1). In other words, Fick's first law is applied in porous media and all the remaining complications related to the description of diffusive transport in a confined pore space are incorporated in the diffusion coefficient $D_{p}$ [34].

Many models have been presented for $D_{p}$, perhaps the simplest being

$D_{p}=\frac{\delta_{D}}{\tau^{2}} D_{0}$

where $D_{0}$ is the intrinsic diffusion coefficient for the solute in a pure solvent, the tortuosity $\tau \geq 1$ includes the effect of complex transport pathways in the pore system while the constrictivity $\delta_{D}<1$ considers variation in pore crosssections along those pathways. Together, the tortuosity and constrictivity define a geometric factor, $\left(\delta_{D} / \tau^{2}\right)<1$, which presents a decrease in diffusivity due to the complex pore geometry. However, appropriate models for $D_{p}$ are still material dependent; that is, a general description for $D_{p}$ remains to be found and $D_{p}$ is thus used below without committing to a specific model.

The advective part of the mass-flux is modeled using $\mathbf{j}_{p, a}=(\epsilon \mathbf{v}) c_{p}$, where $(\epsilon \mathbf{v})$ represents the Darcy velocity expressed in terms of the solvent flow velocity $\mathbf{v}$. Putting all of the above together, one arrives at $[21,35]$

$\partial_{t} c=\nabla \cdot\left(D_{e} \nabla c_{p}-\mathbf{v} c\right)$,

where $D_{e}=\epsilon D_{p}$ is the effective diffusion coefficient. Note that here the diffusion coefficients are all treated as scalars 
simply for ease of notation; in the general formulation, diffusion coefficients are tensors.

A dimensionless form of the model can be written by scaling the related variables using a reference concentration in the solvent $c_{0}$, characteristic length $L$, characteristic velocity $V$, and the intrinsic diffusion coefficient $D_{0}$ :

$\partial_{t}^{*} c *=\nabla^{*} \cdot\left(D_{e}^{*} \nabla^{*} c_{p}^{*}-P e \mathbf{v} c^{*}\right)$.

Dynamic similarity is governed by the Péclect number, $P e=T_{d} / T_{a}$, i.e., the ratio between characteristic time scale for diffusion, $T_{d}=L^{2} / D_{0}$, and advection, $T_{a}=L / V$; Eq. 2 is obtained with the diffusive scaling $t^{*}=t / T_{d}$. Typically in the fracture, where the flow takes place, $T_{a} \ll$ $T_{d}(P e \gg 1)$. At the same time in the matrix, where the flow is negligible, $T_{d} \ll T_{a}(P e \sim 0)$. In other words, a particular computational challenge related to Eq. 2, when considering fracture flows involving matrix-diffusion, is the accommodation of discrepancy in $P e$ between fracture and matrix. Secondly, in heterogeneous materials, the transport parameters, such as $D_{e}$, may vary significantly or may even be discontinuous. This is a fundamental problem for any computational method; the task of finding an acceptable balance between accuracy and lengthy computation times, while avoiding stability issues, can be difficult.

\section{Time domain-random walk method}

Over the years, many approaches have been proposed for the computational modeling of advection-diffusion phenomena. Finite-difference and finite-volume methods can be characterized as the classical approaches. Several alternative methods based on statistical mechanics have also been proposed. For example, a basic random walk method tracks propagation of tracer particles that migrate by jumping at regular intervals: both the length and the direction of a jump are random, but the length depends on the local diffusion coefficient and on the prescribed, regular interval between jumps (aka a constant time step). Tracking the movement of a large collection of these particles reveals their statistical behavior which, when properly simulated, provides an approximate solution for a diffusion equation. That is, the random walk method simulates solute transport by relying on a large number of representative particles each carrying a portion of the solute mass. Advection can be incorporated by including a drift for the jumps.

On the other hand, in the TDRW method, an interesting variant for the treatment of space and time variables is adopted; the particles now jump between sites of a regular grid, or a lattice, and the transition time for a jump is a random variable. That is, here the jump length is fixed by the grid spacing, and the random transition time depends instead on the local diffusion coefficients. A transition probability is assigned for each jump between neighboring lattice sites and these probabilities again depend on the local diffusion coefficients. Advection is considered by introducing a bias on the jump probabilities.

Derivation of the basic random walk method can be achieved using a bottom-up procedure starting from the Langevin equation. This stochastic differential equation is then integrated in time using the Itô-Taylor scheme that provides the usual evolution equation for the random walker [39]. A correspondence between the Fokker-Planck equation (with a modified drift velocity) is next established to finally connect the method with the advection-diffusion equation. At the same time, a top-down procedure can be used to derive the TDRW method. That is, starting from a given macroscopic balance equation, particular TDRW schemes can be constructed in a reverse-engineering fashion.

\subsection{Preparation: semi-discrete finite-volume formulation}

Here, a TDRW scheme is derived from the advectiondiffusion equation in a systematic manner. To account for heterogeneity, or even discontinuity in the transport coefficients involved, it is convenient to start from an integral formulation of the advection-diffusion equation. Namely, following the finite-volume formalism, a volumeaveraged version of Eq. 1 is first written:

$\partial_{t} C=\frac{1}{V} \int_{\Omega} \nabla \cdot\left(D_{e} \nabla c_{p}-\mathbf{v} c\right) \mathrm{d} V=\frac{1}{V} \int_{\Gamma} \mathbf{n} \cdot\left(D_{e} \nabla c_{p}-\mathbf{v} c\right) \mathrm{d} A$,

where $C$ is the volume-averaged concentration and the divergence or Gauss's theorem is applied in the last step. This standard transformation of model equation from differential to integral form relies, ultimately, on the Reynolds transport theorem; the reference or control volumes are here assumed constant with respect to time (i.e., a Eulerian frame of reference is employed). The righthand side accounts for the mass-flux over the surface $\Gamma$ that encloses the reference volume $\Omega$ and $\mathbf{n}$ denotes the unit normal vector (pointing in the outward direction).

Let us assume a complete representation of the spatial domain by a set of non-overlapping volume elements $\Omega_{i}$. Accordingly, Eq. 3 can be expressed in the form

$\partial_{t} C_{i}=\frac{1}{V_{i}} \sum_{j} \int_{\Gamma_{i j}} \mathbf{n} \cdot\left(D_{e} \nabla c_{p}-\mathbf{v} c\right) \mathrm{d} A=-\frac{1}{V_{i}} \sum_{j} A_{i j}\left(J_{i j}^{D}+J_{i j}^{A}\right)$,

where the summation is over the neighboring volume elements $\Omega_{j}$ sharing an interface $\Gamma_{i j}$ with $\Omega_{i}$ while $J_{i j}^{D}$ and $J_{i j}^{A}$ denote surface-averaged mass-flux terms due to diffusion and advection, respectively $\left(A_{i j}\right.$ is the 
area covered by $\Gamma_{i j}$ ). To proceed from here, specific approximations for $J_{i j}^{D}$ and $J_{i j}^{A}$ must be adopted. Namely, this is the point of departure for distinct finite-volume schemes and, consequently, for distinct TDRW schemes.

Here, only cubic grid (or lattice) representations of the spatial domain are considered. Let $\Delta r$ denote the grid spacing which implies $V_{i} \equiv \Delta r^{3}$ and $A_{i j} \equiv \Delta r^{2}$. In addition, all variables related to volumes and surfaces are associated with the centers of cubic cells and square faces, respectively. With these choices, a second-order approximation for the diffusive mass-flux is adopted, that is

$J_{i j}^{D}=-\frac{D_{e, i j}}{\Delta r}\left(\frac{C_{j}}{\epsilon_{j}}-\frac{C_{i}}{\epsilon_{i}}\right)+\mathcal{O}\left(\Delta r^{2}\right)$,

where $D_{e, i j}$ is an appropriately defined effective diffusion coefficient at the center of the face $\Gamma_{i j}$. For example, when the diffusion coefficient is discontinuous exactly at $\Gamma_{i j}$, the harmonic mean $D_{e, i j}=2 D_{e, i} D_{e, j} /\left(D_{e, i}+D_{e, j}\right)$ provides a second-order approximation. Note that $J_{j i}^{D}=-J_{i j}^{D}$ which ensures mass conservation.

A second-order, central approximation is also adopted for the advective mass-flux term:

$J_{i j}^{A}=(\mathbf{n} \cdot \mathbf{v})_{i j} \frac{C_{i}+C_{j}}{2}+\mathcal{O}\left(\Delta r^{2}\right)$.

Clearly $J_{j i}^{A}=-J_{i j}^{A}$. It is well-known that the above secondorder, central-difference approximations here adopted for the diffusive and advective mass-flux terms lead to a stability condition. However, as is later discussed in Section 3.3 and then clearly demonstrated with experiments in Section 4, the emerging second-order accurate TDRW scheme is computationally efficient when considering numerical simulation of fracture flows involving matrixdiffusion despite the stability condition.

In summary, the above mass-flux expressions lead to the differential equations

$d_{t} C_{i}=-B_{i} C_{i}+\sum_{j} B_{j i} C_{j}$,

where

$B_{i}=\sum_{j} B_{i j}, \quad B_{i j}=\frac{D_{p, i j}}{\Delta r^{2}}+\frac{(\mathbf{n} \cdot \mathbf{v})_{i j}}{2 \Delta r}=\frac{D_{0}}{\Delta r^{2}}\left[D_{p, i j}^{*}+P e_{\Delta} \frac{\left(\mathbf{n} \cdot \mathbf{v}^{*}\right)_{i j}}{2}\right]$,

together with $D_{p, i j}:=D_{e, i j} / \epsilon_{i}$, and the grid Péclect number, $P e_{\Delta}:=P e / N$, is defined utilizing the spatial resolution parameter $N=L / \Delta r$.

As an additional observation, note that with square and cubic lattices (at the center of cell $i$ ),

$\sum_{j} \frac{(\mathbf{n} \cdot \mathbf{v})_{i j}}{\Delta r}=(\nabla \cdot \mathbf{v})_{i}+\mathcal{O}\left(\Delta r^{2}\right)$.
Therefore, when considering incompressible fluid flows, where the divergence of the velocity field vanishes, expression for $B_{i}$ simplifies into

$B_{i}=\frac{1}{\Delta r^{2}} \sum_{j} D_{p, i j}$

In any case, the semi-discrete differential equations, Eq. 5 together with Eq. 6, provide the final step for the systematic derivation of a TDRW scheme.

\subsection{Single-particle dynamics}

The ultimate goal is to obtain expressions that describe migration of a random walker, specifically the stochastic dynamics of a single particle. Accordingly, let us consider a configuration with the following point source: the solute mass is concentrated at the center of cell $\Omega_{i}$ and, moreover, $C_{j} \equiv 0$. Then, the solution $C_{i}$ of Eq. 5 is thus given in terms of an exponential function. Namely, for such a configuration, (5) describes exponential decay in the local solute concentration.

The exponential decay can be modeled as a Poisson process, where the interval between successive departure events is an independent identically distributed exponential random variable having a mean $\tau_{i}=1 / B_{i}$ (the variance is $\tau_{i}^{2}$ ). Here, the intervals are identified as transition times during which a random walker advances from site $i$ to a neighboring site $j$. Since the transition times are exponentially distributed, appropriate random numbers can be generated using the inverse transform sampling method:

$t_{i \rightarrow j}=-\tau_{i} \ln u$,

where $u$ is a random number from the standard uniform distribution. Note that the transition times are sampled without any consideration to which of the neighbors the random walker will advance next. Based on Eq. 7, when considering incompressible fluid flows, the mean transition time, $\tau_{i}=1 / B_{i}$, does not depend on advective transport.

The probability of a random walk transition to a particular neighbor is now considered. Note first that the right-hand side of Eq. 5 denotes the net flux of mass over the faces of cell $\Omega_{i}$. This net flux is the difference between the mass-fluxes out of and into the cell $\Omega_{i}$. In more detail, $J_{i \rightarrow j}=\Delta r B_{i j} C_{i}$ represents the flux of mass from the cell $\Omega_{i}$ into the cell $\Omega_{j}$ per unit area and per unit time (the massflux in the reverse direction is similarly given by $\Delta r B_{j i} C_{j}$ ). From this perspective, it is quite natural to associate the probability of a random walk transition from the site $i$ to a 
neighboring site $j$ with the corresponding relative mass-flux out of the cell $\Omega_{i}$. Specifically,

$p_{i \rightarrow j}=\frac{J_{i \rightarrow j}}{\sum_{j} J_{i \rightarrow j}}=\tau_{i} B_{i j}$.

To summarize, the migration of a tracer particle in the derived TDRW scheme is described by Eqs. 6, 8, and 9. In detail, the transport of tracer particles can be modeled using the following algorithm.

1. Construct the transition probability matrix according to Eq. 9. Size of the matrix for a cubic grid is $s_{x} \times s_{y} \times s_{z} \times s_{w}$ (here, the number of jump directions per grid site, $s_{w}$, is assumed constant across the grid).

2. Determine the total number of particles $N_{\text {tot }}$ to be simulated. For each particle, follow the next steps.

3. Randomly select the initial location of a particle from the set of grid sites configured as allowed initial locations.

4. Determine the direction of a jump according to a random number and the transition probability matrix constructed at step 1 . Here, sampling of a single random number from the standard uniform distribution is required to determine the jumping direction among the possible directions.

5. Determine the transition time $t_{i \rightarrow j}$ for the jump according to Eq. 8. Here, sampling of a single random number from the standard uniform distribution is required.

6. Check if the particle has reached the configured target area. If no, return to step 4. If yes, record the total time elapsed $t_{\mathrm{tot}}=\sum t_{i \rightarrow j}$.

7. Check if the simulation has been performed for the required number of particles. If no, return to step 3 .

\subsection{Numerical properties}

Positive transition probabilities are guaranteed when $B_{i j} \geq$ 0. Based on Eqs. 6 and 9, this means that locally and in each direction, the condition

$P e_{\Delta} \leq \frac{2 D_{p, i j}^{*}}{\left|\mathbf{n} \cdot \mathbf{v}^{*}\right|_{i j}}$

must be fulfilled. This condition emerges from the second-order, central-difference approximation adopted for the advective mass-flux term; it is well-known that a similar restriction in central-difference-based finitevolume schemes is required for guaranteeing stability of a simulation. If Eq. 10 is not respected, the behavior of the TDRW scheme is ill-defined and consequently nothing can be expected from the computed results. Thus, to further emphasize this critical aspect, in case (10) is violated, the behavior of the scheme is arbitrary or rather depends on the specific implementation choices; therefore, the computed solutions are not guaranteed to have any relation to the corresponding theoretical solutions of the original modeling equation.

The condition in Eq. 10 excludes simulations of mass transfer in pure advection cases. However, as these cases do not belong to the current problem statement, this restriction is here dismissed. Furthermore, the condition is always fulfilled in pure diffusion regions (a typical scenario, for example, in the porous media surrounding the fracture). Therefore, here the condition matters in the fracture, or flow channels, and must be respected by tuning the simulation parameters appropriately. Often, the fracture presents a totally open flow domain (that is $\epsilon=1$, implying $D_{p, i j}^{*}=$ 1). If one further assumes that the flow field (in reduced units) is set up such that $\left|\mathbf{n} \cdot \mathbf{v}^{*}\right|_{i j} \leq 1$ holds everywhere, the condition for guaranteeing positive transition probabilities is simply $\mathrm{Pe}_{\Delta} \leq 2$.

Recently, a simulation of advection-diffusion phenomena with the TDRW method was proposed based on an upwind finite-difference approximation of the advective mass-flux $[13,31,38]$; this was essentially the same approximation proposed by Flamm et al. [11] as an extension to the LKMC method. With the current notation, the corresponding upwind finite-volume approximation leads to

$$
B_{i j}=\frac{D_{p, i j}}{\Delta r^{2}}+\frac{1}{\Delta r} \max \left[(\mathbf{n} \cdot \mathbf{v})_{i j}, 0\right] .
$$

Clearly, this expression would provide unconditionally positive probabilities. However, it is known that the first-order accurate upwind schemes introduce severe numerical diffusion especially when simulating configurations involving large concentration gradients. Therefore, simulation of mass transfer in fracture flows together with, for example, release of tracer pulses into complex flow channels would be problematic. In fact, this presents the main motivation for the second-order, central-difference-based TDRW scheme proposed here. Very recently and for the same reasons, Lee and Sinno [22] suggested an improved treatment of advective fluxes in the LKMC method. Specifically, they chose to employ an approximation, known as the exponential scheme in the finite-volume context, which suffers less from the numerical diffusion but is not guaranteed to provide second-order accuracy in general.

It is also important to note that in the TDRW method, there is no independent control of the time resolution. Namely, there is no discrete time step degree of freedom contrary to many other computational methods. To clarify, let us consider the incompressible fluid flow case. The local mean transition time defined by Eq. 7 implies

$\tau_{i} \sim \Delta r^{2} / D_{0}=T_{d} / N^{2}$.

That is, the mean transition time, an analogue to a discrete time step, is controlled by the intrinsic diffusion 
coefficient and the grid spacing, evidently representing a diffusive scaling of the time resolution. In the case of pure diffusion, the value of $D_{0}$ automatically adjusts the mean transition time but does not affect the transition probabilities. Hence, the number of particle jumps required to travel a given distance remains constant (assuming a given lattice, namely $\Delta r$ is not altered). Accordingly, in this simplistic setup, simulations with different $D_{0}$ are computationally equally demanding, thus reflecting the constant jump length determined by a fixed $\Delta r$. It is then instructive to compare the situation with the standard random walk method where maintaining a constant jump length requires explicitly tuning the discrete time step simulation parameter. When the diffusion coefficient exhibits significant spatial variation, this difference can be of considerable relevance.

Furthermore, let us consider a problem scenario where $P e$ is fixed, alongside $L$ and geometrical features in general, but $V$ and $D_{0}$ remain free for tuning a computationally favorable simulation. Based on Eqs. 6 and 9 and since the ratio $V / D_{0}$ is now fixed, the bias in local transition probabilities towards the flow direction can be controlled only via the grid spacing (the bias is directly proportional to $\Delta r$, or $1 / N)$. A smaller bias means that the propagation of a tracer particle is more erratic; it is thus likely that more particle jumps are needed to travel a given distance along a flow path. Therefore, to summarize the above, the computational resources needed to execute a simulation with the TDRW method are heavily dependent on the spatial resolution or grid spacing.

Finally, just increasing the number of tracer particles per simulation cannot be used to achieve an arbitrary accuracy in computed solutions with a fixed grid spacing. That is, the limitations set by a given grid spacing cannot be avoided, and the particle count is primarily used for controlling fluctuations in the simulated results; projection and filtering techniques can also be used for suppressing fluctuations (see for example Ref. [39] and references therein). Fortunately, due to the statistical nature of the method, raising the particle count is a task ideally suited for parallel processing increasingly relied upon by modern computational resources. Moreover, the number of tracer particles can be increased on demand, that is, a proper particle count does not need to be known a priori to successfully execute a simulation. This feature facilitates a frugal usage of computational resources. This also further distinguishes the TDRW method from classical computational methods (such as the FD and FV methods), for which an analogous control option is not available.

The power of the presented top-down derivation of TDRW schemes, in contrast to a bottom-up derivation (relying on a microscopic particle perspective), is that the whole numerical machinery already developed, for example, for the corresponding finite-volume schemes, are directly available for exploitation. For example, the current grid Péclect number limitation could perhaps be relieved by developing a TDRW scheme based on an appropriate total variation diminishing scheme, a topic left for further investigation.

Moreover, when a TDRW scheme is derived based on a finite-volume formalism, particles can jump to any of the neighboring sites (i.e., to the centers of the neighboring cells). To keep the treatment accessible, we derived above a TDRW scheme for cubic grids where the particles always jump along the Cartesian coordinate directions. In general, however, each cell has a specific set of possible jump directions and this set depends on the neighboring cell configuration (i.e., on the spatial discretization of simulation domain with finite volumes). Thus, an analysis focusing on how the (cell-dependent) set of possible jump directions influence numerical properties of a TDRW scheme becomes a relevant research topic. As a related matter, utilizing isotropic approximations of differential operators [23, 32] in the derivation of TDRW schemes is another possible future direction for development. Finally, Noetinger et al. [31] attempted to connect the TDRW method with the continuous time-random walk method. This connection, its theoretical foundations, and the possible prospects it introduces also deserve further investigation.

\section{Numerical experiments}

The constructed TDRW scheme was validated below by comparing numerical results against analytical solutions. The proposed second-order accurate, central-difference scheme was also compared with the first-order accurate upwind scheme in one simple flow configuration. The benchmark systems considered involve a rectangular flow channel connected with a porous matrix on one side of the flow channel (in the simplest cases, porosity is zero). At the beginning of the simulations, the particles were released from a single plane in the flow channel and caught in another plane at the end of the channel. The channel was also extended backwards from the particle release plane to allow unrestricted, diffusive spreading of the solute pulse.

Figure 1 shows a schematic of the benchmark system used. In case of $\epsilon=0$ (that is without the porous matrix), all simulations considered below are performed in a flow channel of length $\left(L_{x}\right) 1000 \mathrm{~mm}$, height (a) $20 \mathrm{~mm}$, width $\left(L_{z}\right) 100 \mathrm{~mm}$, and using voxel size $(\Delta r)$ of $1 \mathrm{~mm}$. In the case of permeable porous matrix (that is $\epsilon>0$ ), the simulation setups remain the same, except that the channel width is halved ( $L_{z}=50 \mathrm{~mm}$ ) to save computer memory and the porous matrix height $\left(L_{y}\right)$ is $100 \mathrm{~mm}$. On a HP Z840 workstation (256 GB of memory, 2 INTEL Core i7-6900K 


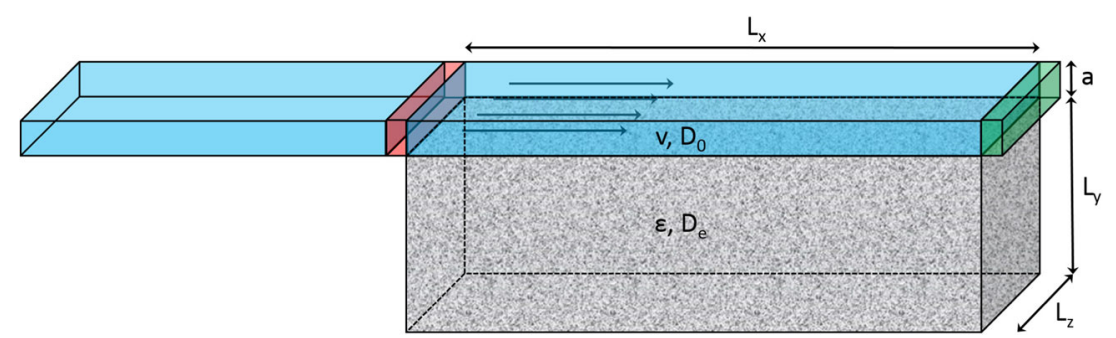

Fig. 1 A schematic of the geometry used for verification simulations. The flow channel (advection velocity $v$, diffusion coefficient $D_{0}$, length $L_{x}$, height $a$, width $L_{z}$ ) is shown in light blue and the porous matrix (porosity $\epsilon$, effective diffusion coefficient $D_{e}$, height $L_{y}$ ) is in

processors with 32 threads in total), our naive, unoptimized MATLAB implementation ran for $8.0 \times 10^{-5}$ and $3.2 \times 10^{-3}$ $\mathrm{s}$ per particle in a rectangular channel without porous matrix and with flow velocities of $1 \times 10^{-6} \mathrm{~m} / \mathrm{s}$ and $5 \times 10^{-8} \mathrm{~m} / \mathrm{s}$, respectively. The porous matrix increased the calculation time by approximately $10 \%$.

\subsection{Flow in a rectangular channel}

The simplest simulations are first performed using a constant uniform flow velocity in a rectangular channel with impermeable walls on all sides. The aim is to verify that diffusion spreads the pulse correctly and that the mean breakthrough time is correct. In this case, the analytical solution for the breakthrough curve at the end of the flow channel is [18]

$C\left(\tau_{t}\right)=\frac{1}{\mu \sqrt{\pi \tau_{t}^{3}}} \exp \left[-\frac{1}{4 \mu^{2}}\left(\tau_{t}+\frac{1}{\tau_{t}}-2\right)\right]$,

where $\tau_{t}=t Q / V_{c}, t$ is the time, $Q$ is the flow rate in the channel, $V_{c}$ is the flow channel volume, $\mu=$ contact with one side of the flow channel. The particle release and target areas are shown in red and green, respectively (arrows mark direction of the flow)

$\sqrt{D_{0} / L_{x} v}, D_{0}$ is the intrinsic diffusion coefficient in the solvent, $L_{x}$ is the length of the flow channel, and $v$ is the flow velocity in the channel. The simulations were performed using $5 \times 10^{6}$ particles, and two values for the intrinsic diffusion coefficient, $2 \times 10^{-9} \mathrm{~m}^{2} / \mathrm{s}(\sim$ water $)$ and $2 \times 10^{-8} \mathrm{~m}^{2} / \mathrm{s}$; the flow velocities used ranged from $5 \times 10^{-8} \mathrm{~m} / \mathrm{s}$ to $1 \times 10^{-6} \mathrm{~m} / \mathrm{s}$ which is close to the upper limit defined by Eq. 10, when $D_{0}=2 \times 10^{-9} \mathrm{~m}^{2} / \mathrm{s}$. The resulting breakthrough curves, shown in Fig. 2, were in close accordance with the curves produced by the analytical solutions with the same parameters. The simulation results are presented as time-dependent particle fluxes through target area (fluxes normalized using the total number of particles).

Continuing with the same flow configuration, the proposed second-order accurate, central-difference scheme was then compared with the first-order accurate upwind scheme. To this end, breakthrough curves with $D_{0}=2 \times$ $10^{-9} \mathrm{~m}^{2} / \mathrm{s}, 5 \times 10^{6}$ particles, and flow velocities from $5 \times 10^{-6}$ to $1 \times 10^{-8} \mathrm{~m} / \mathrm{s}$ are shown in Fig. 3. Furthermore, the Euclidian norm $\left(\ell^{2}\right)$ of the relative error was determined
Fig. 2 Simulated results using the proposed TDRW scheme in a rectangular flow channel with impermeable walls, four values for the constant flow velocity $(v)$, and two values for the intrinsic diffusion coefficient $\left(D_{0}\right)$. Solid lines present the corresponding analytical solutions, Eq. 11. The results are reported as particle flux through target area and are normalized using the total number of particles

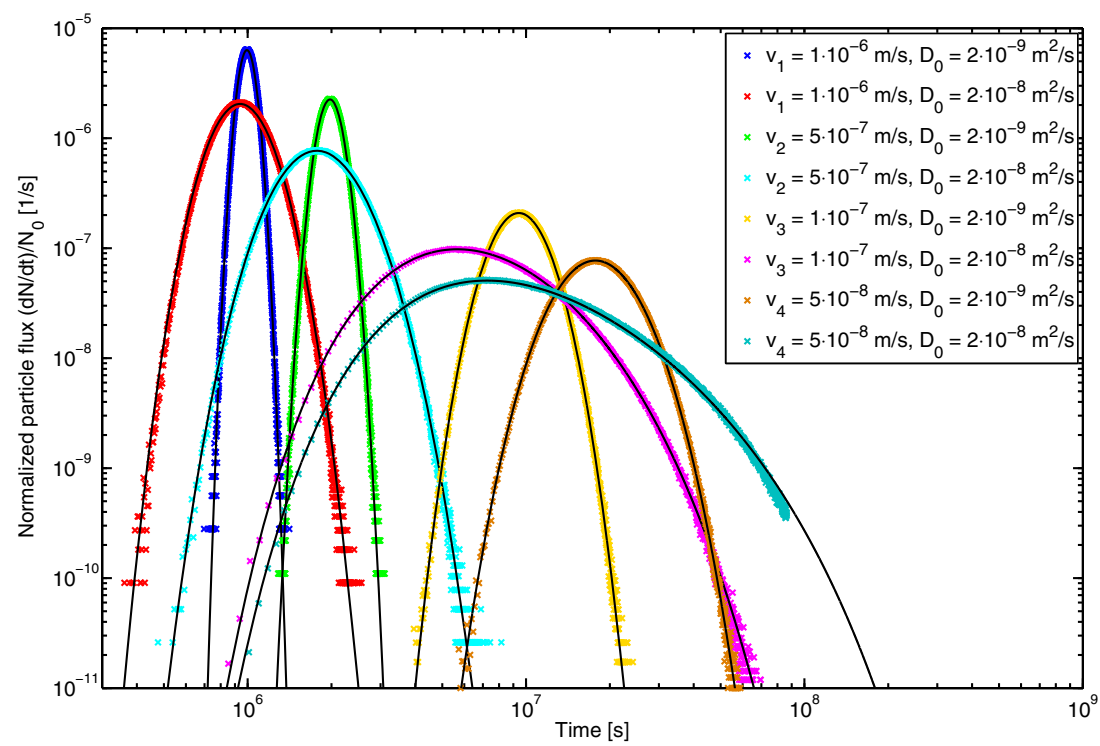



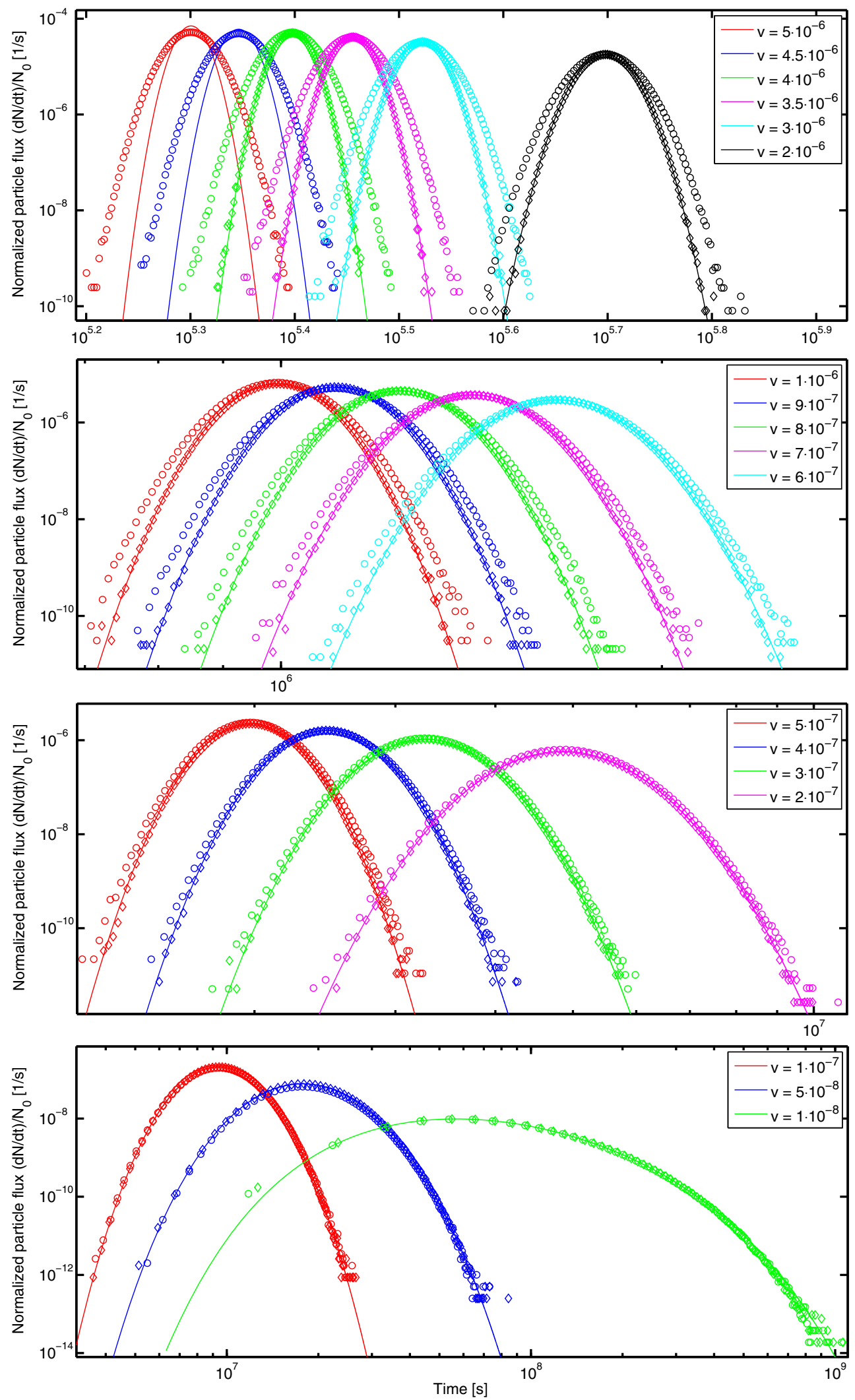

Fig. 3 Comparison of breakthrough curves in a rectangular channel with constant flow velocities and $D_{0}=2 \times 10^{-9} \mathrm{~m}^{2} / \mathrm{s}$ : centraldifference (diamond) and upwind (circle) results and analytical solutions (Eq. 11, solid line). The central-difference results are in a close agreement with the analytical solutions when $P e_{\Delta} \leq 2(v \leq 4 \times$ $\left.10^{-6} \mathrm{~m} / \mathrm{s}\right)$, whereas the upwind results reach a similar agreement only when $P e_{\Delta} \leq 0.05\left(v \leq 1 \times 10^{-7} \mathrm{~m} / \mathrm{s}\right)$ (see also Table 1$)$ 
Table 1 Euclidian norm $\left(\ell^{2}\right)$ of the relative errors for central-difference and upwind results in a rectangular channel with constant flow velocities (that is, various grid Péclect numbers, $\left.P e_{\Delta}\right)$ and $D_{0}=2 \times 10^{-9} \mathrm{~m}^{2} / \mathrm{s}$; the relative errors are computed with respect to the analytical solution, Eq. 11

\begin{tabular}{llll}
\hline Flow velocity, $v\left[\frac{\mathrm{m}}{\mathrm{s}}\right]$ & $P e_{\Delta}[-]$ & Central-difference, $\ell^{2}[-]$ & Upwind, $\ell^{2}[-]$ \\
\hline $5 \times 10^{-6}$ & 2.50 & - & $1.6 \times 10^{6}$ \\
$4.5 \times 10^{-6}$ & 2.25 & - & $3.5 \times 10^{5}$ \\
$4.0 \times 10^{-6}$ & 2.00 & 2.0 & $2.1 \times 10^{5}$ \\
$3.5 \times 10^{-6}$ & 1.75 & 1.8 & $3.1 \times 10^{4}$ \\
$3.0 \times 10^{-6}$ & 1.50 & 1.5 & $6.6 \times 10^{3}$ \\
$2.0 \times 10^{-6}$ & 1.00 & 1.0 & 418 \\
$1.0 \times 10^{-6}$ & 0.50 & 1.4 & 51 \\
$9.0 \times 10^{-7}$ & 0.45 & 0.7 & 29 \\
$8.0 \times 10^{-7}$ & 0.40 & 0.7 & 20 \\
$7.0 \times 10^{-7}$ & 0.35 & 1.3 & 18 \\
$6.0 \times 10^{-7}$ & 0.30 & 0.9 & 18 \\
$5.0 \times 10^{-7}$ & 0.25 & 0.9 & 6.7 \\
$4.0 \times 10^{-7}$ & 0.20 & 0.9 & 6.2 \\
$3.0 \times 10^{-7}$ & 0.15 & 1.1 & 4.7 \\
$2.0 \times 10^{-7}$ & 0.10 & 0.5 & 1.6 \\
$1.0 \times 10^{-7}$ & 0.05 & 0.9 & 1.4 \\
$5.0 \times 10^{-8}$ & 0.025 & 1.3 & 2.4 \\
$1.0 \times 10^{-8}$ & 0.005 & 1.9 & \\
\hline
\end{tabular}

A small value for $\ell^{2}$ means a small deviation between the numerical approximation and analytical solution for each of the simulated breakthrough curves to quantify deviations from the corresponding analytical solutions (see Table 1). In this numerical analysis, time intervals with less than five particles arriving at the target area were not considered in order to decrease the effect of numerical fluctuation caused by insufficient statistics.

The central-difference results are in a good agreement with analytical solutions when $P e_{\Delta} \leq 2$ and, moreover, the agreement seems to be practically independent of flow velocity. On the other hand, velocities greater than $4 \times$ $10^{-6} \mathrm{~m} / \mathrm{s}$ impose $P e_{\Delta}>2$ and the central-difference scheme breaks down (as predicted by the condition ensuring positive transition probabilities, Eq. 10). The upwind results reach a similar agreement only when $P e_{\Delta} \leq 0.05(v \leq 1 \times$ $10^{-7} \mathrm{~m} / \mathrm{s}$ ); Table 1 shows that with faster velocities, the error grows rapidly. Based on Fig. 3, peaks of the breakthrough curves are in the right position with the upwind scheme but the shape of the curves is wider than the analytical solution predicts. By reducing the voxel size, $\Delta r$, the accuracy of the upwind scheme with higher velocities can be improved, but this is a computationally expensive option.

In the second benchmark system, a more realistic flow field was introduced into the channel. Here, a parabolic flow profile between two plates,

$v(y)=6 v_{\text {ave }}\left(\frac{1}{4}-\frac{y^{2}}{a^{2}}\right)$,

was used, where $v_{\text {ave }}$ is the average flow velocity and $y$ is the vertical distance from center of the channel ( $a$ is the channel height). Now, in addition to diffusional spreading, differences in local flow velocities cause extra spreading of the pulse. Such spreading is called Taylor dispersion and can be accounted for in the analytical solution, Eq. 11, by replacing $D_{0}$ appearing in $\mu$ with

$\widehat{D}_{0}=D_{0}\left(1+\frac{1}{192} \frac{a^{2} v_{\text {ave }}^{2}}{D_{0}^{2}}\right)$.

This setup was simulated using $5 \times 10^{6}$ particles, four values for the average velocity $\left(1 \times 10^{-6}, 5 \times 10^{-7}\right.$, $1 \times 10^{-7}$, and $5 \times 10^{-8} \mathrm{~m} / \mathrm{s}$ ) and two values for the intrinsic diffusion coefficient, $2 \times 10^{-9} \mathrm{~m}^{2} / \mathrm{s}$ ( $\sim$ water) and $2 \times 10^{-8} \mathrm{~m}^{2} / \mathrm{s}$. These simulation parameters cover a wide range of (grid) Péclect numbers while respecting the condition in Eq. 10. The resulting breakthrough curves from the simulations, together with the corresponding curves by analytical solutions, are presented in Fig. 4. It can be seen that when $D_{0}$ is increased, the breakthrough curve is spread and median arrival time is decreased as the transport by diffusion becomes more dominant. With the smallest $v_{\text {ave }}$ used, the change in median arrival time becomes most visible as the change in ratio of $v_{\text {ave }}$ and particle speed by diffusion is largest. In general, the simulated results are in a good agreement with the analytical solutions.

\subsection{Flow in a rectangular channel connected with a porous matrix}

A porous matrix is next included on one face of the flow channel, as shown in Fig. 1. Now, the analytical solution for the breakthrough curve at the end of the channel becomes more complex. It can, however, be derived 
Fig. 4 Simulated results using the proposed TDRW scheme in a rectangular flow channel with impermeable walls, parabolic flow profiles specified by four average velocities ( $v_{\text {ave }}$ ), and with two values for the intrinsic diffusion coefficient $\left(D_{0}\right)$. Solid lines represent the corresponding analytical solutions, Eq. 11, where the modified diffusion coefficient, Eq. 13, is used in place of $D_{0}$ accounting for Taylor dispersion. The results are reported as particle flux through target area and are normalized using the total number of particles

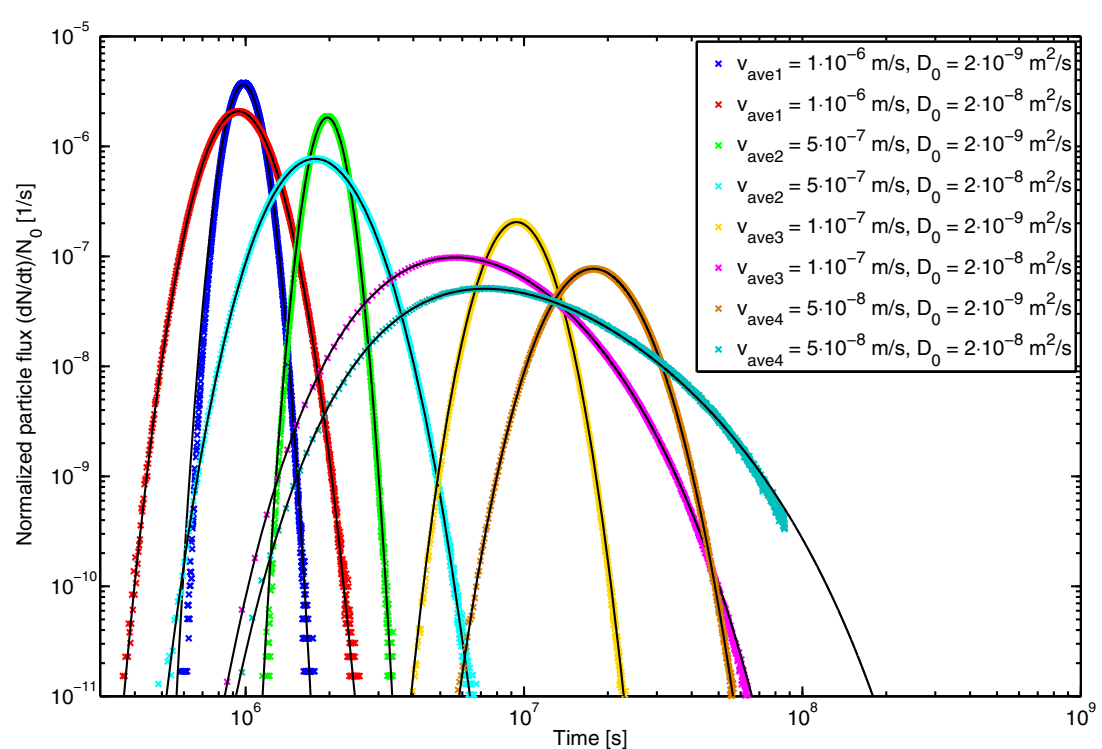

using a dimensionless representation for partial differential equations, Laplace conversion, and reordering the terms. The analytical solution for the breakthrough curve in this case and its derivation can be found in Ref. Kekäläinen et al. [18]. The simulations were run with the same flow channel dimensions as in the previous cases, except the channel width $\left(L_{z}\right)$ was decreased to $50 \mathrm{~mm}$ to reduce memory usage. The height of the porous matrix $\left(L_{y}\right)$ is $100 \mathrm{~mm}$. The range of constant velocities used was the same as before, the matrix porosity was $1 \%$, and the amount of particles was $5 \times 10^{7}$. In the channel, the intrinsic diffusion coefficient of water was used $\left(D_{0}=2 \times 10^{-9} \mathrm{~m}^{2} / \mathrm{s}\right)$. Furthermore, two values for the effective diffusion coefficients in the matrix were used. The values of the porosity and the effective diffusion coefficients for the matrix were chosen according to typical values in nature and in geological formations [19]. Note that there is no advection in the porous matrix.

The results of these simulations agree closely with the analytical solutions and are shown in Fig. 5. The main peak of a breakthrough curve forms as in the case without the diffusive matrix since most of the particles travel through the system without interacting with the matrix. The particles that have entered the matrix arrive later on average, and thus the effect of the matrix can be seen in the late part of the breakthrough curve (tails of the curves). Comparison of breakthrough curves from simulations using the same $v$ but different $D_{e}$ shows the difference in interaction rate of particles with the matrix and the effect of matrix finity. As particles in the flow channel jump more likely into a neighboring site with a high $D_{e}$ than one with a low $D_{e}$, the
Fig. 5 Simulated results using the proposed TDRW scheme in a rectangular flow channel (here $L_{z}=50 \mathrm{~mm}$ ) connected with an adjacent diffusive matrix $(\epsilon=1 \%)$ along with the analytical solutions by Kekäläinen et al. [18] (solid lines). Four values for the constant flow velocities $(v)$ and two values for the effective diffusion coefficients $\left(D_{e}\right)$ were used together with the intrinsic diffusion coefficient

$D_{0}=2 \times 10^{-9} \mathrm{~m}^{2} / \mathrm{s}$. The

simulation results are reported as particle flux through target area and are normalized using total number of particles

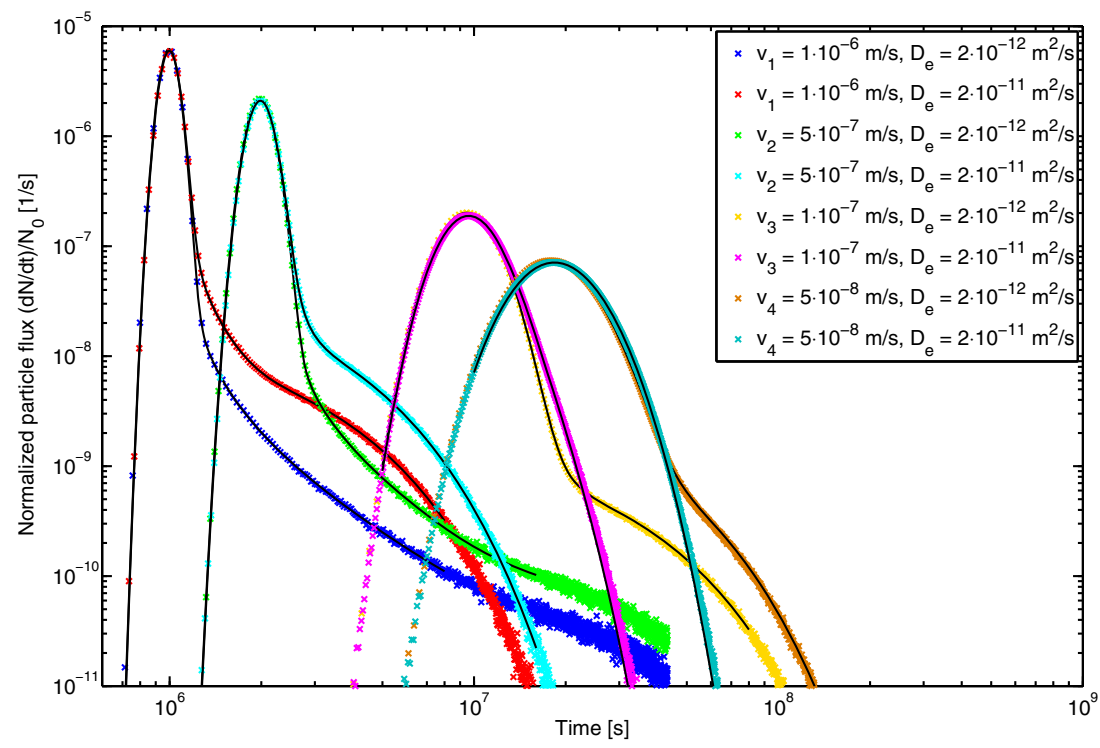


Fig. 6 Simulated results using the proposed TDRW scheme in a rectangular flow channel (here $L_{z}=50 \mathrm{~mm}$ ) connected with an adjacent diffusive matrix $(\epsilon=1 \%)$ along with the analytical solutions by Kekäläinen et al. [18] (solid lines). Four values for the average velocity of the parabolic flow profile $\left(v_{\text {ave }}\right)$ and two values for the effective diffusion coefficient $\left(D_{e}\right)$ were used together with the intrinsic diffusion coefficient $D_{0}=2 \times 10^{-9} \mathrm{~m}^{2} / \mathrm{s}$. The simulation results are reported as particle flux through target area and are normalized using total number of particles

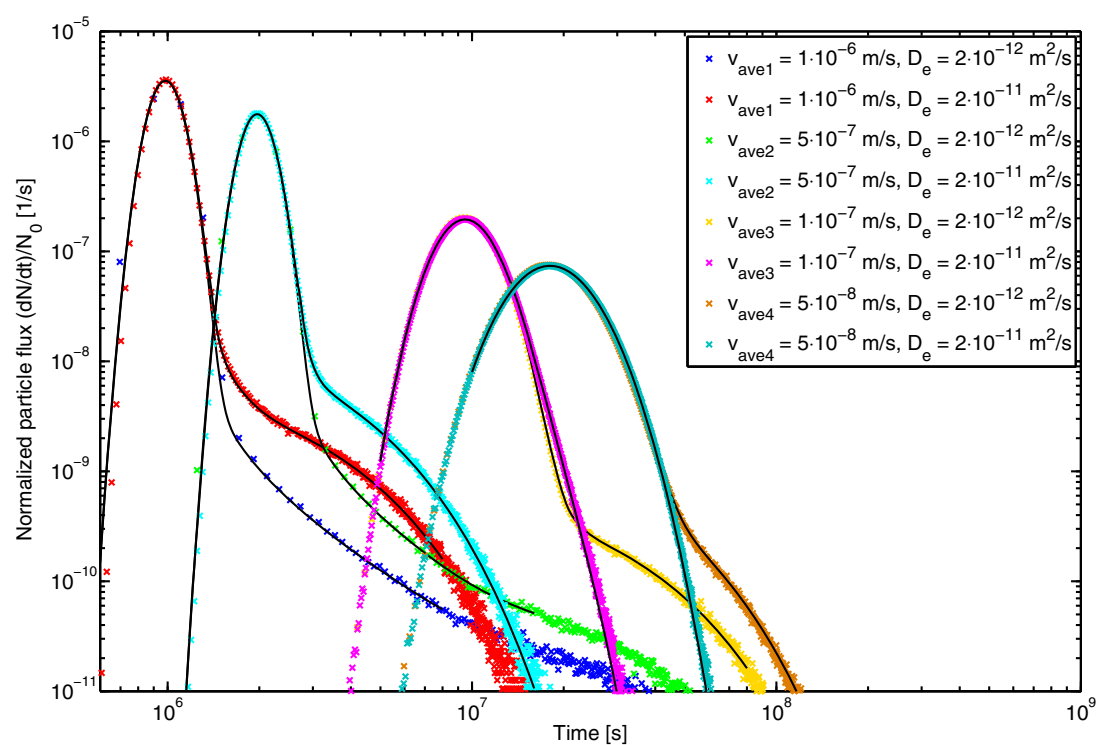

tail of the breakthrough curve starts to develop earlier in the case of a high $D_{e}$. The finity of the matrix can be seen in the shape of the tail, as particles are reflected from the end of the matrix. This effect causes the formation of a hump next to the advection-dominated part. Moreover, the particle flux approaches zero rapidly after the hump. When finity of the matrix is not dominating in the late part of the breakthrough curves, a slowly decreasing tail is formed. In the cases with the slowest flow rates $\left(1 \times 10^{-7}\right.$ and $\left.5 \times 10^{-8} \mathrm{~m} / \mathrm{s}\right)$ and with $D_{e}$ of $2 \times 10^{-11} \mathrm{~m}^{2} / \mathrm{s}$, the hump has merged to the main peak and matrix diffusion has deformed the shape of main peak.

Finally, the parabolic flow profile, Eq. 12, was used in simulations that include the diffusive matrix. Taylor dispersion was again included in the analytical solution by replacing $D_{0}$ with $\widehat{D}_{0}$ of Eq. 13. Four different average velocities and two different effective diffusion coefficients were used together with the intrinsic diffusion coefficient $D_{0}=2 \times 10^{-9} \mathrm{~m}^{2} / \mathrm{s}$ and $5 \times 10^{7}$ particles. The results, shown in Fig. 6, agree very well with the analytical solutions. The breakthrough curves show a similar shape as in the case with constant $v$. However, since the differences in local velocities create additional dispersion, the advection-dominated mean peaks of the breakthrough curves are wider in this case.

\section{Conclusions}

The aim of this study was to incorporate accurate treatment of advective fluxes into the TDRW method and to verify that the resulting scheme can correctly model, firstly, the spreading of a tracer pulse by diffusion and Taylor dispersion transported in simple fracture flow and, secondly, systems involving fracture flows coupled with matrix diffusion. Accordingly, a central-differencebased TDRW scheme was derived in a reverse-engineering fashion, starting from an appropriate advection-diffusion equation and then following a systematic discretization procedure to arrive at the equations governing a singleparticle random walk on a lattice. The adopted top-down derivation of TDRW schemes is powerful in the sense that the whole numerical machinery already developed for the corresponding finite-difference or finite-volume schemes are directly available for exploitation.

Several benchmark cases were then simulated to validate the proposed scheme; by varying the simulation parameters, a wide range of (grid) Péclect numbers were covered in each case. The numerical results were then compared against corresponding analytical solutions. While it is noteworthy that analytical solutions are available even for the fracture flows coupled with matrix diffusion, they do not come in a simple, closed form such as the solutions for the more elementary benchmark cases. Very good agreement between the numerical and analytical solutions was observed in general and we thus concluded that the constructed scheme behaves according to the theoretical predictions. Furthermore, it was shown that the second-order accurate central-difference TDRW scheme considerably improves the reliability of the TDRW scheme for modeling advective flow in comparison with the previously constructed firstorder accurate upwind TDRW scheme. The difference between these schemes with regard to the central-difference scheme was found to be considerable when (grid) Péclect was from 0.1 to 2 .

With the TDRW method, many problems that affect other numerical methods can be avoided, including stability issues and particularly small discrete time steps. On the 
other hand, the TDRW method may require tracking a relatively larger number of random walks to properly capture statistics from the low-porosity regions as well. However, modern computers rely increasingly on parallel processing and hence pushing the computational burden into the number of independent random walks seems advantageous. Another limitation of the TDRW method is that it does not allow independent control of the time resolution; it relies instead on a diffusive scaling of the time variable (the time resolution is fixed by the grid spacing and the intrinsic diffusion coefficient). It is important to note that a given regular grid or lattice determines the best spatial accuracy achievable, and the number of particles is mainly used for suppressing fluctuations in the computed solutions.

Nevertheless, the TDRW method is an attractive alternative for computational modeling of mass transfer in fracture flows coupled with matrix diffusion, a problem that intrinsically involves multiple relevant scales. Finally, there remain several possible topics for future method development, including utilization of total variation diminishing schemes and isotropic approximations of differential operators in the TDRW framework. In principle, the method is capable of modeling mass transfer in small-scale heterogeneous fracture networks. However, further studies for real heterogeneous fractures and comparison with previously developed methods are still necessary. The up-scaling of the method to field scale will be challenging due to the restriction given in Eq. 10. However, in the future, the TDRW method could be used to verify the mathematical expressions for the interaction of solutes in the fracture and porous matrix used in field-scale models.

Funding information Financial support from the Finnish Research Programme on Nuclear Waste Management (KYT2018) is gratefully acknowledged.

Open Access This article is distributed under the terms of the Creative Commons Attribution 4.0 International License (http:// creativecommons.org/licenses/by/4.0/), which permits unrestricted use, distribution, and reproduction in any medium, provided you give appropriate credit to the original author(s) and the source, provide a link to the Creative Commons license, and indicate if changes were made.

\section{References}

1. Aromaa, H., Voutilainen, M., Ikonen, J., Yli-Kaila, M., Poteri, A., Siitari-Kauppi, M.: Through diffusion experiments to study the diffusion and sorption of HTO, 36Cl, 133Ba and 134Cs in crystalline rock. J. Contam. Hydrol. 222, 101-111 (2019). https://doi. org/10.1016/j.jconhyd.2019.03.002

2. Berkowitz, B., Kosakowski, G., Margolin, G., Scher, H.: Application of continuous time random walk theory to tracer test measurements in fractured and heterogeneous porous media. Ground. Water. 39(4), 593-604 (2001). https://doi.org/10.1111/j. 1745-6584.2001.tb02347.x
3. Bodin, J., Delay, F., de Marsily, G.: Solute transport in a single fracture with negligible matrix permeability: 1. Fundamental mechanisms. Hydrogeol. J. 11(4), 418-433 (2003). https://doi.org/ 10.1007/s10040-003-0268-2

4. Bortz, A., Kalos, M., Lebowitz, J.: A new algorithm for Monte Carlo simulation of Ising spin systems. J. Comput. Phys. 17(1), 10-18 (1975). https://doi.org/10.1016/0021-9991(75)90060-1

5. Cvetkovic, V., Frampton, A.: Transport and retention from single to multiple fractures in crystalline rock at äspö (Sweden): 2 . Fracture network simulations and generic retention model. Water Resour. Res. 46(5), w05506 (2010). https://doi.org/10.1029/2009 WR008030

6. Delay, F., Porel, G.: Inverse modeling in the time domain for solving diffusion in a heterogeneous rock matrix. Geophys. Res. Lett. 30, 1147-1150 (2003). https://doi.org/10.1029/2002GL016428

7. Delay, F., Porel, G., Sardini, P.: Modelling diffusion in a heterogeneous rock matrix with a time-domain Lagrangian method and an inversion procedure. C. R. Geosci. 334(13), 967973 (2002). https://doi.org/10.1016/S1631-0713(02)01835-7

8. Dentz, M., Scher, H., Holder, D., Berkowitz, B.: Transport behavior of coupled continuous-time random walks. Phys. Rev. E. 78(4), 041,110 (2008). https://doi.org/10.1103/PhysRevE.78.041110

9. Dentz, M., Gouze, P., Russian, A., Dweik, J., Delay, F.: Diffusion and trapping in heterogeneous media: An inhomogeneous continuous time random walk approach. Adv. Water. Resour. 15(1), 13-22 (2012). https://doi.org/10.1016/j.advwatres.2012.07.015

10. Farah, N., Delorme, M., Ding, D.Y., Wu, Y.S., Bossie Codreanu, D.: Flow modelling of unconventional shale reservoirs using a DFM-MINC proximity function. J. Petrol. Sci. Eng. 173, 222-236 (2019). https://doi.org/10.1016/j.petrol.2018.10.014

11. Flamm, M., Diamond, S., Sinno, T.: Lattice kinetic Monte Carlo simulations of convective-diffusive systems. J. Chem. Phys. 130(9), 094,904 (2009). https://doi.org/10.1063/1.3078518

12. Foster, S.: The chalk groundwater tritium anomaly - a possible explanation. J. Hydrolog. 25(1), 159-165 (1975). https://doi.org/ 10.1016/0022-1694(75)90045-1

13. Gjetvaj, F., Russian, A., Gouze, P., Dentz, M.: Dual control of flow field heterogeneity and immobile porosity on non-Fickian transport in Berea sandstone. Water. Resour. Res. 51(10), 82738293 (2013). https://doi.org/10.1002/2015WR017645

14. Haggerty, R., Gorelick, S.M.: Multiple-rate mass transfer for modeling diffusion and surface reactions in media with pore-scale heterogeneity. Water Resour. Res. 31(10), 2383-2400 (1995). https://doi.org/10.1029/95WR10583. wR10583

15. Ikonen, J., Voutilainen, M., Söderlund, M., Jokelainen, L., SiitariKauppi, M., Martin, A.: Sorption and diffusion of selenium oxyanions in granitic rocks. J. Cont. Hydrol. 192, 203-211 (2016). https://doi.org/10.1016/j.jconhyd.2016.08.003

16. Ikonen, J., Sardini, P., Siitari-Kauppi, M., Martin, A.: In situ migration of tritiated water and iodine in Grimsel granodiorite, part II: Assessment of the diffusion coefficients by TDD modelling. J. Radioanal. Nucl. Chem. 311(1), 339-348 (2017). https://doi.org/ 10.1007/s10967-016-5041-9

17. Kekäläinen, P.: Analytical solutions to matrix diffusion problems. AIP Conf. Proc. 1618(1), 513-516 (2014). https://doi.org/10.10 63/1.4897787

18. Kekäläinen, P., Voutilainen, M., Poteri, A., Hölttä, P., Hautojärvi, A., Timonen, J.: Solutions to and validation of matrixdiffusion models. Transp. Porous. Med. 87(1), 125-149 (2011). https://doi.org/10.1007/s11242-010-9672-y

19. Kuva, J., Voutilainen, M., Kekäläinen, P., Siitari-Kauppi, M., Timonen, J., Koskinen, L.: Gas phase measurements of porosity, diffusion coefficient, and permeability in rock samples from Olkiluoto bedrock, Finland. Transp. Porous. Med. 107(1), 187204 (2015). https://doi.org/10.1007/s11242-014-0432-2 
20. Kuva, J., Voutilainen, M., Kekäläinen, P., Siitari-Kauppi, M., Sammaljärvi, J., Timonen, J., Koskinen, L.: Gas phase measurements of matrix diffusion in rock samples from Olkiluoto bedrock, Finland. Transp. Porous. Med. 115(1), 1-20 (2016). https://doi.org/10.1007/s11242-016-0748-1

21. LaBolle, E., Quastel, J., Fogg, G.: Diffusion theory for transport in porous media: Transition-probability densities of diffusion processes corresponding to advection-dispersion equations. Water. Resour. Res. 34(7), 1685-1693 (1998). https://doi.org/10.1029/98 WR00319

22. Lee, Y., Sinno, T.: Analysis of the lattice kinetic Monte Carlo method in systems with external fields. J. Chem. Phys. 145(23), 234,104 (2016). https://doi.org/10.1063/1.4972052

23. Mattila, K., Hegele, J.r., L., Philippi, P.: High-accuracy approximation of high-rank derivatives: Isotropic finite differences based on lattice-Boltzmann stencils. Sci. World. J 142, 907 (2014). https://doi.org/10.1155/2014/142907

24. McCarthy, J.: Continuous-time random walks on random media. J. Phys. A-Math. Gen. 26(11), 2495-2503 (1993). https://doi.org/ 10.1088/0305-4470/26/11/004

25. McDermott, C., Walsh, R., Mettier, R., Kosakowski, G., Kolditz, O.: Hybrid analytical and finite element numerical modeling of mass and heat transport in fractured rocks with matrix diffusion. Comput. Geosci. 13(3), 349-361 (2009). https://doi.org/10.1007/ s10596-008-9123-9

26. Montroll, E., Weiss, G.: Random walks on lattices. II. J. Math. Phys. 6(2), 167-181 (1965). https://doi.org/10.1063/1.1704269

27. Neretnieks, I.: Diffusion in the rock matrix: An important factor in radionuclide retardation? J. Geophys. Res. 85(B8), 4379-4397 (1980). https://doi.org/10.1029/JB085iB08p04379

28. Nœtinger, B., Estebenet, T.: Up-scaling of double porosity fractured media using continuous-time random walks methods. Transp. Porous Med. 39, 315-337 (2000). https://doi.org/10.1023/ A: 1006639025910

29. Nœtinger, B., Estebenet, T., Landereau, P.: A direct determination of the transient exchange term of fractured media using a continuous time random walk method. Transp. Porous Med. 44, 539-557 (2001). https://doi.org/10.1023/A:1010647108341

30. Nœtinger, B., Estebenet, T., Quintard, M.: Up-scaling flow in fractured media: equivalence between the large scale averaging theory and the continuous time random walk method. Transp. Porous Med. 43, 581-596 (2001). https://doi.org/10.1023/A:1010733724498

31. Noetinger, B., Roubinet, D., Russian, A., Borgne, T.L., Delay, F., Dentz, M., de Dreuzy, J., Gouze, P.: Random walk methods for modeling hydrodynamic transport in porous and fractured media from pore to reservoir scale. Transp. Porous. Med. 115(2), 345-385 (2016). https://doi.org/10.1007/s11242-016-0693-z

32. Patra, M., Karttunen, M.: Stencils with isotropic discretization error for differential operators. Numer. Meth. Part. Differ. Equ. 22(4), 936-953 (2006). https://doi.org/10.1002/num.20129

33. Posiva: Safety case for the disposal of spent nuclear fuel at olkiluoto - Models and data for the repository system 2012. Tech. rep., Posiva Oy (2003)

34. Rasilainen, K.: Matrix diffusion model: In situ tests using natural analogue. PhD thesis, Helsinki University of Technology, Finland (1997)

35. Rhodes, M.: Transport in Heterogeneous Porous Media. PhD thesis. Imperial College, London (2008)

36. Robinet, J., Sardini, P., Delay, F., Hellmuth, K.H.: The effect of rock matrix heterogeneities near fracture walls on the residence time distribution (RTD) of solutes. Transp. Porous. Med. 72(3), 393-408 (2008). https://doi.org/10.1007/s11242-007-9159-7
37. Robinet, J., Sardini, P., Coelho, D., Parneix, J., Prêt, D. Sammartino, S., Boller, E., Altmann, S.: Effects of mineral distribution at mesoscopic scale on solute diffusion in a clay-rich rock: Example of the Callovo-Oxfordian mudstone (Bure, France). Water. Resour. Res. 48(5), W05,554 (2012). https://doi.org/10.1029/2011WR011352

38. Russian, A., Dentz, M., P Gouze, P.: Time domain random walks for hydrodynamic transport in heterogeneous media. Water. Resour. Res. 52(5), 3309-3323 (2016). https://doi.org/10.1002/2015WR018511

39. Salamon, P., Fernàndez-Garcia, D., Gómez-Hernández, J.: A review and numerical assessment of the random walk particle tracking method. J. Contam. Hydrol. 87(3-4), 277-305 (2006). https://doi.org/10.1016/j.jconhyd.2006.05.005

40. Sardini, P., Robinet, J., Siitari-Kauppi, M., Delay, F., Hellmuth, K.H.: Direct simulation of heterogeneous diffusion and inversion procedure applied to an out-diffusion experiment. Test case of Palmottu granite. J. Contam. Hydrol. 93(1-4), 21-37 (2007). https://doi.org/10.1016/j.jconhyd.2007.01.011

41. Stumm, W.: Chemistry of the Soil-Water Interface: Processes at the Mineral-Water and Particle-Water Interface in Natural Systems. Wiley, New York (1992)

42. Svensson, U., Löfgren, M., Trinchero, P., Selroos, J.O.: Modelling the diffusion-available pore space of an unaltered granitic rock matrix using a micro-DFN approach. J. Hydrol. 559, 182-191 (2018). https://doi.org/10.1016/j.jhydrol.2018.02.041

43. Tang, D., Frind, E., Sudicky, E.: Contaminant transport in fractured porous media: Analytical solutions for a single fracture. Water. Resour. Res. 17(3), 555-564 (1981). https://doi.org/10.1029/WR017i003p00555

44. Tecklenburg, J., Neuweiler, I., Carrera, J., Dentz, M.: Multi-rate mass transfer modeling of two-phase flow in highly heterogeneous fractured and porous media. Adv. Water. Resour. 91, 63-77 (2016). https://doi.org/10.1016/j.advwatres.2016.02.010

45. Toivanen, J., Mattila, K., Hyväluoma, J., Kekäläinen, P., Puurtinen, T., Timonen, J.: Simulation software for flow of fluid with suspended point particles in complex domains: Application to matrix diffusion. Lect. Notes Comput. Sc. 7782, 434-445 (2013). https://doi.org/10.1007/978-3-642-36803-5_32

46. Toivanen, J., Mattila, K., Puurtinen, T., Timonen, J.: LBM simulations of matrix diffusion with sorption. AIP Conf. Proc. 1618(1), 517-520 (2014). https://doi.org/10.1063/1.4897788

47. Trinchero, P., Molinero, J., Deissmann, G., Svensson, U., Gylling, B., Ebrahimi, H., Hammond, G., Bosbach, D., Puigdomenech, I.: Implications of grain-scale mineralogical heterogeneity for radionuclide transport in fractured media. Transp. Porous. Med. 116(1), 73-90 (2017). https://doi.org/10.1007/s11242-016-0765-0

48. Voutilainen, M., Kekäläinen, P., Hautojärvi, A., Timonen, J.: Validation of matrix diffusion modeling. Phys. Chem. Earth. 35(68), 259-264 (2010). https://doi.org/10.1016/j.pce.2010.04.005

49. Voutilainen, M., Sardini, P., Siitari-Kauppi, M., Kekäläinen, P., Aho, V., Myllys, M., Timonen, J.: Simulated diffusion of tracer in altered tonalite with heterogeneous distribution of porosity. Transp. Porous. Med. 96(2), 319-336 (2013). https://doi.org/10.1007/s11242-012-0090-1

50. Voutilainen, M., Poteri, A., Helariutta, K., Siitari-Kauppi, M., Nilsson, K., Andersson, P., Byegård, J., Skålberg, M., Kekäläinen, P., Timonen, J., Lindberg, A., Pitkänen, P., Kemppainen, K., Liimatainen, J., Hautojärvi, A., Koskinen, L.: In-situ experiments for investigating the retention properties of rock matrix in ONKALO, Olkiluoto, Finland. In: Proceedings of the Annual Waste Management Symposium (WM2014), March 2-6, pp. 1969-1981, Phoenix (2014) 
51. Voutilainen, M., Kekäläinen, P., Poteri, A., Siitari-Kauppi, M., Helariutta, K., Andersson, P., Nilsson, K., Byegård, J., Skålberg, M., Yli-Kaila, M., Koskinen, L.: Comparison of water phase diffusion experiments in laboratory and in situ conditions. J. Hydrol. 575, 716-729 (2019)

52. Voutilainen, M., Kekäläinen, P., Siitari-Kauppi, M., Sardini, P., Muuri, E., Timonen, J., Martin, A.: Modeling transport of cesium in Grimsel granodiorite with micrometer scale heterogeneities and dynamic update of $K_{d}$. Water. Resour. Res. 53(11), 9245-9265 (2017). https://doi.org/10.1002/2017WR020695

53. Yan, B., Mi, L., Chai, Z., Wang, Y., Killough, J.E.: An enhanced discrete fracture network model for multiphase flow in fractured reservoirs. J. Petrol. Sci. Eng. 161, 667-682 (2017). https://doi.org/10.1016/j.petrol.2017.12.016

Publisher's note Springer Nature remains neutral with regard to jurisdictional claims in published maps and institutional affiliations. 